\title{
Mathematical model of an electric car to diagnose its traction qualities on a chassis dynamometer
}

\author{
Aleksandr Fedotov', Oleg Yan'kov', and Anton Chernyshkov ${ }^{1 *}$ \\ ${ }^{1}$ Department of Automobile Transport, Irkutsk National Research Technical University, 83 \\ Lermontov str., Irkutsk, 664074, Russia
}

\begin{abstract}
The paper outlines the developed mathematical model of an electric vehicle to control its traction and dynamic qualities on a chassis dynamometer. The purpose of the work is to expand the capabilities of diagnostics and analytical determination of electric vehicle dynamics parameters. The developed mathematical model includes descriptions of the following processes: the operation of the electric motor, torsional vibrations in the transmission of an electric car and the chassis dynamometer, the processes of tires interaction with the circular-shaped surfaces of the chassis dynamometer.
\end{abstract}

\section{Introduction}

In present time, more and more electric cars are on the roads of the modern world. Experience suggests that their number will only grow in the future. It means that the issues of their maintaining, restoring and providing high-quality diagnostics along with monitoring their traction and dynamic qualities will become especially relevant.

Chassis dynamometers provide the most effective control of traction and dynamic qualities. They are one of the most informative tools to monitor the traction properties of vehicles under operating conditions. When conducting diagnostics of vehicles on chassis dynamometers processes occur that affect the measurement of parameters of traction and dynamic qualities. Such processes include torsional vibrations in the chassis dynamometer transmission, power losses in the elements of the chassis dynamometer transmission.

Currently, there is a need to expand the capabilities of diagnostics and analytical determination of the parameters of traction and dynamic properties of electric vehicles. For this purpose, a mathematical model of the process of functioning of electric vehicles on a hybrid chassis dynamometer of the IRNTU design was developed [1, 2, 3, 4]. The model includes descriptions of the processes: the functioning of the electric motor, torsional vibrations and power losses in the transmission of the electric car and the chassis dynamometer, the processes of interaction of tires with the circular-shaped surfaces of the chassis dynamometer.

* Corresponding author: antonchernyshkov@,gmail.com 


\section{Results and Discussion}

The process of functioning of an electric motor is described by the functional dependence of the torque on its output shaft on the speed of its rotation:

$$
M_{e}=M_{e \max }\left(a_{e} \cdot \operatorname{arctg}\left(b_{e} \frac{n_{e}}{n_{e \max }}-c_{e}\right)-d_{e}\left(\frac{n_{e}}{n_{e \max }}\right)^{a_{e 1}}-b_{e 1}\left(\frac{n_{e}}{n_{e \max }}\right)^{c_{e 1}}+d_{e 1}\right)
$$

where $M_{e \max }$ - maximum torque of the electric motor; $n_{e}-$ motor rotor speed; $n_{\text {emax }}-$ maximum speed of the motor rotor; $a_{e}, b_{e}, c_{e}, d_{e}, a_{e l}, b_{e l}, c_{e l}, d_{e l}$ - approximation coefficients.

In this case, the power on the motor shaft was determined by the formula:

$$
N_{e}=\frac{M_{e} n_{e}}{9550}
$$

The speed of rotation of the motor rotor was determined from the formula:

$$
n_{e}=\frac{30 \cdot \omega_{e}}{\pi}
$$

where $\omega_{e}-$ angular velocity of the drive wheel.

To calculate the torsional vibrations in the transmission of an electric car during its acceleration on the chassis dynamometer a kinematic scheme was drawn up (Figure 1). The kinematic scheme takes into account: the moments of inertia of the electric motor $I^{\prime}{ }_{1}$, the gearbox and differential $I_{2}^{\prime}$, the driving wheels $I_{3}^{\prime}$, the running valves $I_{4}^{\prime}$ and the flywheel masses of the chassis dynamometer $I^{\prime}{ }_{5}$, the stiffness of the shafts $c^{\prime}{ }_{1}, \mathrm{c}^{\prime}{ }_{2}$, the semi-axles $c^{\prime}{ }_{3}$, the tires $\mathrm{c}^{\prime}{ }_{4}$ and the chain transmission $c^{\prime}{ }_{5}$. Also, when calculating the mathematical model, such parameters as the gear ratios of the electric vehicle IT and the chain transmission, the realized coupling moment $M_{f}$, and the rolling resistance moment $M_{\varphi}$ are taken into account.

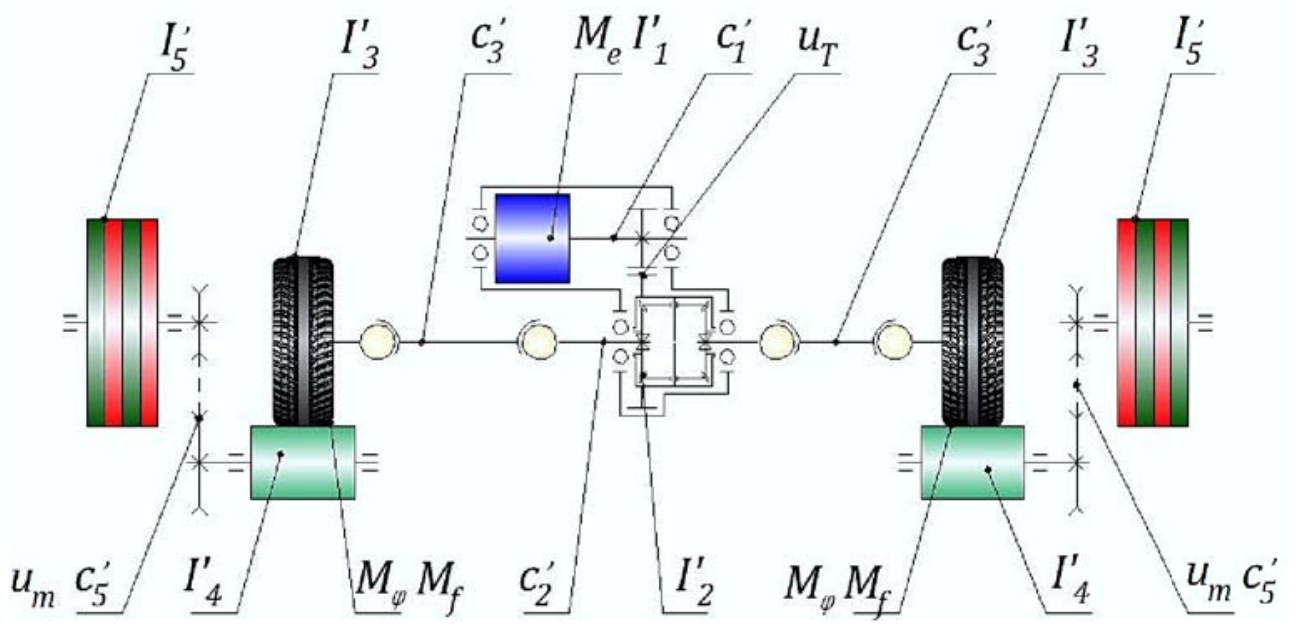

Fig. 1. Kinematic scheme for calculating torsional vibrations.

The presented kinematic scheme (Figure 1) was transformed into a partial calculation scheme (Figure 2)that is divided into sections which take into account the moments of inertia of the transmission elements: $I_{1}$ - the moment of inertia of the electric motor and the input shaft of the gearbox; $I_{2}$ - the moment of inertia of the output shaft of the gearbox and the 
differential; $I_{3}$ - the moment of inertia of the half - axle and the wheel; $I_{4}$ - the moment of inertia of the chassis dynamometer; $I_{5}$ - the moment of inertia of the flywheel masses of the chassis dynamometer. Formally, such a transformation corresponds to a linear reduction of the coordinates of the kinematic scheme of the model the main condition of which is the conservation of kinetic and potential energy [1,3].

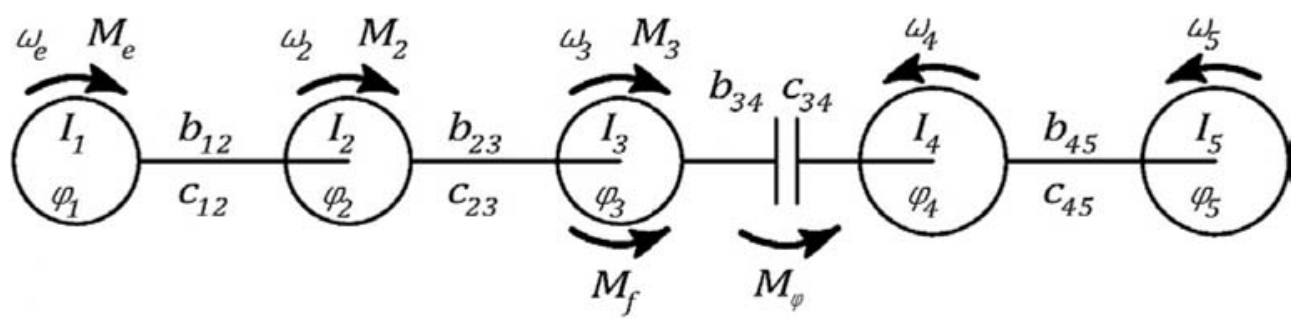

Fig. 2. Partial design scheme for describing torsional vibrations.

In the mathematical model, it is currently assumed that the moments of inertia of the semiaxles and wheels $I_{3}$, the chassis dynamometers $I_{4}$ and the main masses $I_{5}$, located on the left and right sides, are equal in magnitude. Moreover, the stiffness and damping coefficients of the shafts located on the left and right sides relative to the longitudinal axis of the chassis dynamometer are assumed to be equal in value. In general, the moments of inertia in the partial scheme are calculated taking into account the transfer ratios $[1,3]$ :

$$
I_{1}=I_{1}^{\prime} ; \quad I_{2}=\frac{I_{2}^{\prime}}{u_{T}} ; \quad I_{3}=I_{3}^{\prime} ; \quad I_{4}=I_{4}^{\prime} ; \quad I_{5}=\frac{I_{5}^{\prime}}{u_{M}} ;
$$

where $u_{M}$ - the gear ratio between the chassis dynamometer and the flywheel masses; $I_{l}$ - moment of inertia of the electric motor and the input shaft of the gearbox; $I_{2}$ - moment of inertia of the output shaft of the gearbox and differential; $I_{3}$ - moment of inertia of the halfaxle and wheel; $I_{4}$ - the moment of inertia of the running reels; $I_{5}$ - moment of inertia of the flywheel masses of the chassis dynamometer.

The calculation of torsional vibrations is implemented by a system of differential equations describing the dynamics of rotation of the elements of the trans-mission and the chassis dynamometer.

The dynamics of rotation of the motor rotor is described by the following differential equations:

$$
\begin{aligned}
\frac{d^{2} \varphi_{1}}{d t^{2}} & =\frac{M_{e}}{I_{1}+I_{2}+2 I_{3}} \\
\frac{d \varphi_{1}}{d t} & =\frac{d \varphi_{1}}{d t}+\frac{d^{2} \varphi_{1}}{d t^{2}} d t \\
\varphi_{1} & =\varphi_{1}+\frac{d \varphi_{1}}{d t} d t
\end{aligned}
$$

where $\varphi_{l}$ - angle of rotation of the motor rotor.

The dynamics of rotation of the differential housing is realized by the following differential equations: 


$$
\begin{gathered}
\frac{d^{2} \varphi_{2}}{d t^{2}}=\frac{c_{12}\left(\varphi_{1}-\varphi_{2}\right)-c_{23}\left(\varphi_{2}-\varphi_{3}\right)-b_{12} \frac{d \varphi_{2}}{d t}}{I_{2}} \\
\frac{d \varphi_{2}}{d t}=\frac{d \varphi_{2}}{d t}+\frac{d^{2} \varphi_{2}}{d t^{2}} d t \\
\varphi_{2}=\varphi_{2}+\frac{d \varphi_{2}}{d t} d t
\end{gathered}
$$

where $\varphi_{2}$ - differential rotation angle; $\varphi_{3}$ - angle of rotation of the driving wheel of an electric car; $b_{12}$ - damping coefficient of the shaft connecting the electric motor and the gearbox; $c_{12}$ - shaft stiffness between electric motor and gearbox; $c_{23}$ - half-axle stiffness.

The dynamics of rotation of the driving wheels of an electric car is realized by the following differential equations:

$$
\begin{gathered}
\frac{d^{2} \varphi_{3}}{d t^{2}}=\frac{c_{23}\left(\varphi_{2}-\varphi_{3}\right)-c_{34}\left(\varphi_{3}-\varphi_{4}\right)-b_{23} \frac{d \varphi_{3}}{d t}-F_{f} r_{k 0}}{I_{3}} \\
\frac{d \varphi_{3}}{d t}=\frac{d \varphi_{3}}{d t}+\frac{d^{2} \varphi_{3}}{d t^{2}} d t \\
\varphi_{3}=\varphi_{3}+\frac{d \varphi_{3}}{d t} d t
\end{gathered}
$$

where $\varphi_{4}$ - angle of rotation of the running drum; $b_{23}$ - half-axis damping coefficient; $c_{34}$ - wheel stiffness.

The transmission of torque from the driving wheel to the running bars and flywheel masses of the stand and vice versa is possible only when there are longitudinal reactions between the tire and the support surfaces of the chassis dynamometer, the value of which is limited by the realized clutch torque.

$$
R_{x 1 i}=\varphi_{\max } R_{z 1 i} f(s)
$$

where $\varphi_{\max }$ - maximum coefficient of adhesion; $R_{\mathrm{z} 1 \mathrm{i}}$ - the normal reaction of the chassis dynamometers to the action of a normal load on the driving wheel of an electric car.

The dynamics of rotation of the running reels of the chassis dynamometer is realized by the following differential equations:

$$
\begin{aligned}
\frac{d^{2} \varphi_{4}}{d t^{2}} & =\frac{R_{x 1} \cdot r_{\sigma \sigma}-F_{f} \cdot r_{k 0}}{I_{4}} ; \\
\frac{d \varphi_{4}}{d t} & =\frac{d \varphi_{4}}{d t}+\frac{d^{2} \varphi_{4}}{d t^{2}} d t \\
\varphi_{4} & =\varphi_{4}+\frac{d \varphi_{4}}{d t} d t
\end{aligned}
$$

where $\varphi_{5}$ - angle of rotation of the flywheel masses; $b_{45}$ - damping coefficient of the chain transmission between the chassis dynamometer and the flywheel masses; $F_{f}$-rolling resistance force; $f$ - rolling resistance coefficient; $R_{x l}$ - longitudinal reaction acting in the 
contact spot of the driving wheels with the chassis dynamometers; $r_{k 0}$ - rolling radius of the wheel; $r_{\sigma \sigma}$ - chassis dynamometer radius.

The dynamics of rotation of the flywheel masses of the chassis dynamometer is described by the following differential equations:

$$
\begin{gathered}
\frac{d^{2} \varphi_{5}}{d t^{2}}=\frac{c_{45}\left(\varphi_{4}-\varphi_{5}\right)-b_{45} \frac{d \varphi_{5}}{d t}}{I_{5}} \\
\frac{d \varphi_{5}}{d t}=\frac{d \varphi_{5}}{d t}+\frac{d^{2} \varphi_{5}}{d t^{2}} d t \\
\varphi_{5}=\varphi_{5}+\frac{d \varphi_{5}}{d t} d t
\end{gathered}
$$

where $c_{45}$ - stiffness of the chain transmission between the chassis dynamometer and the flywheel masses.

The calculated scheme of the force interaction of the tire with the cylindrical surfaces of the chassis dynamometers during the acceleration of the driving wheels of the electric car on the chassis dynamometer is shown in Figure 3. To determine the longitudinal reactions of $R_{x I i}$ on wheels, a model of a wheel with an elastic tire was used, developed by A. B. Dick and based on the normalized $f(s)$ diagram $[5,6]$ :

$$
f(s)=\sin (A \cdot \operatorname{arctg}(B \cdot S))
$$

where $A, B$ - coefficients that characterize the shape of the $f(s)$ diagram; $S$-slippage defined from the expression $(22)[5,6]$.

$$
S=1-\frac{\omega_{6 \sigma} r_{6 \sigma}}{\omega_{k} r_{k 0}}
$$

where $\omega_{б б}-$ angular velocity of the chassis dynamometer.

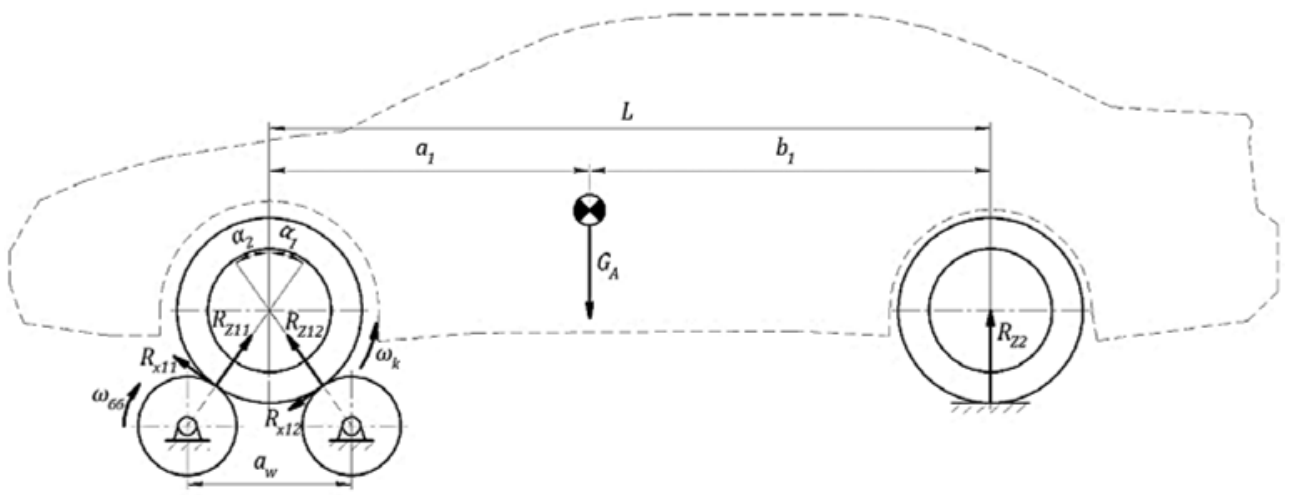

Fig. 3. Calculation scheme of forces in the interaction of the wheel with the chassis dynamometer.

The change in the rolling radius of the wheel, which occurs due to the redistribution of the load on the axles during acceleration, is taken into account by the formula (23):

$$
r_{k 0 i}=r_{c}-R_{z i} \cdot c_{2}-\sqrt{R_{z i} \cdot c_{1}} ;
$$

where $c_{1}, c_{2}$ - constants; $R_{z i}$ - normal reaction of the $i^{\text {th }}$ axis. 
The weight distribution on the front and rear axles of an electric car is determined from the formulae:

$$
\begin{aligned}
& G_{k 1}=\frac{G_{a} b_{1}}{L} ; \\
& G_{k 2}=\frac{G_{a} a_{1}}{L} ;
\end{aligned}
$$

where $G_{a}$ - electric vehicle weight; $a_{l}, b_{1}$ - distances from the center of mass of the electric vehicle to the front and rear axles, respectively; $L$ - wheelbase of an electric car.

The normal reactions on the front axle wheels to the running drums surfaces of the treadmills are calculated by the formula (26):

$$
R_{z 1 i}=\sqrt{\frac{G_{k 1}^{2}}{2+4 \operatorname{Cos}\left(\alpha_{1}+\alpha_{2}\right)}} ;
$$

where $\alpha_{1}$ - angle between vectors $G_{k l}$ and $R_{z 11} ; \alpha_{2}$ - angle between vectors $G_{k 1}$ and $R_{z 12}$.

The maximum realized torque on the clutch $M_{\varphi}$ can differ from the torque supplied to the wheel and is found from the expression (27):

$$
M_{\varphi}=R_{x} r_{k 0}
$$

The rolling resistance moment of the wheel $M_{f}$ is found from the expression (28):

$$
M_{f}=R_{z} r_{k 0} f
$$

The mathematical model of car acceleration is implemented in the Delphi programming environment. All differential equations were solved by the numerical Euler method with an integration step of $\Delta \mathrm{t}=0.00001 \mathrm{~s}$. The results of the calculations of the developed mathematical model are presented in Figure 4.

To confirm the results of the calculation of torsional vibrations according to the developed mathematical model it is necessary to conduct an experimental study. For this purpose, a system was made to measure the signal from the ABS sensor of an electric car. The developed system is installed in the standard connectors of the electric car without damaging the electrical wiring and without distorting the sensor signals. 


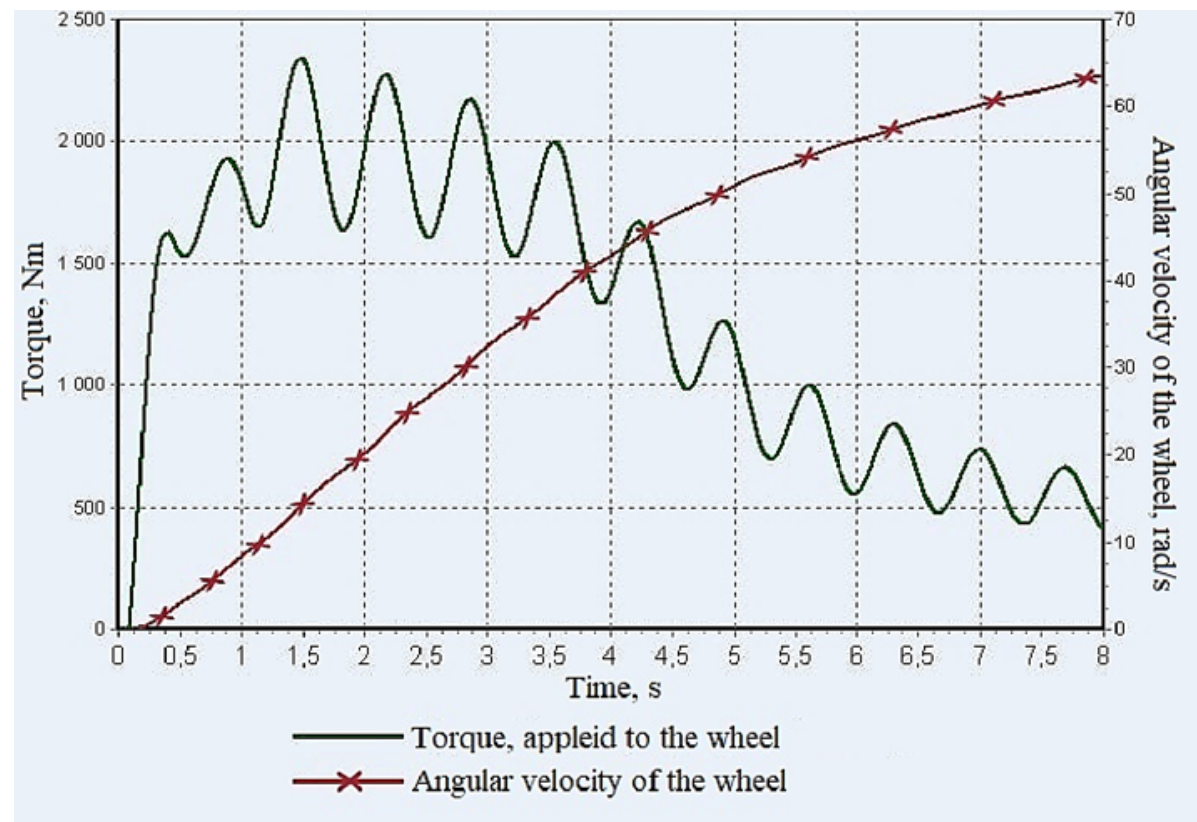

Fig. 4. Graph of fluctuations in the torque applied to the wheel of an electric car.

The measured signal from the ABS sensor of each drive wheel goes to the signal amplifier, then to the frequency-voltage converter, and then goes through the ADC to the personal computer. Based on the indicators of the ABS sensors, one can track the angular speed of the driving wheels of the vehicle. It is also possible to calculate the angular acceleration of the wheel that allows you to determine the fluctuations of the torque supplied to the wheel. Figure 5 shows a comparison of the results of an experimental study and mathematical calculations of torsional vibrations during acceleration of an electric car. 


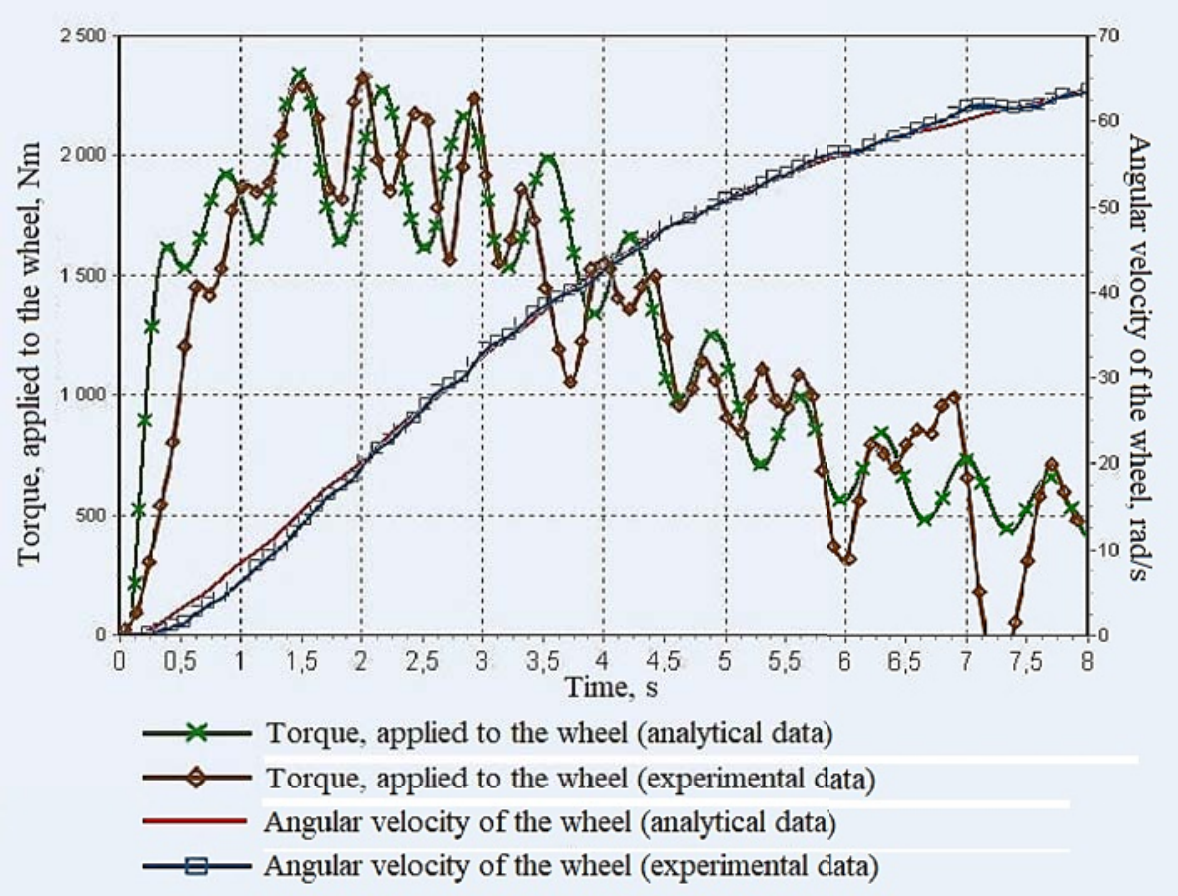

Fig. 5. Comparison of the results of an experimental study and mathematical calculations of torsional vibrations during acceleration of an electric motor.

\section{Conclusion}

A mathematical model of the acceleration process of an electric car on the chassis dynamometer is developed, which takes into account such processes as: the operation of the electric motor, torsional vibrations in the transmission of the electric car and the chassis dynamometer, the processes of interaction of tires with the cylindrical surfaces of chassis dynamometers. The mathematical model allows calculating such output parameters such as: the torque of the electric motor, the traction force on the wheels of the electric car, the angular velocities of the transmission elements of the electric car and the chassis dynamometer, the torques, twisting shafts of the transmission of the electric car, the acceleration time of the electric car, etc.

In the future, the authors plan to expand the capabilities of the mathematical model, taking into account the impact of the electrodynamic brake on the chassis dynamometer, which creates a force of resistance to the movement of the electric car. It is also planned to add the calculation of power and speed losses in the elastic tires of the driving wheels.

\section{References}

1. A I Fedotov, O S Yankov and A S Chernyshkov Traction control and diagnostics of electric and unmanned vehicles on roller stands E3S Web of Conferences Khabarovsk: EDP Sciences, P. 01021 (2020)

2. A I Fedotov, O S Yankov and A S Chernyshkov Russia Patent №199093 (2020)

3. A I Fedotov, O S Yankov and A S Chernyshkov Russia Patent №2021613529 (2021) 
4. O S Yankov and A S Chernyshkov Design development of force-measuring magnetostrictive sensor of brake tester Proc. Int. Conf. Russia pp 53-59 (2018)

5. A I Fedotov Vehicle Diagnostics Textbook for university students on Operation of transport and technological machines and complexes (Irkutsk: Publishing House of ISTU) p 476

6. A B Dick On the radius of the elastic wheel Automobile industry No. 10. pp. 21-28 (2020) 